\title{
Seroprevalence of Measles, Rubella, Tetanus, and Diphtheria Antibodies among Children in Haiti, 2017
}

\author{
Anna A. Minta, ${ }^{1 *}$ Jocelyne Andre-Alboth, ${ }^{2}$ Lana Childs, ${ }^{1,3}$ Douglas Nace, ${ }^{4}$ Gloria Rey-Benito, ${ }^{5}$ Jacques Boncy, ${ }^{2}$ Paul Adrien, ${ }^{6}$ \\ Jeannot Francois, ${ }^{7}$ Nadia Phaïmyr Jn Charles, ${ }^{8}$ Valery Blot, ${ }^{9}$ Jodi Vanden Eng, ${ }^{1}$ Jeffrey W. Priest, ${ }^{10}$ Eric Rogier, ${ }^{4}$ \\ and Rania A. Tohme ${ }^{1}$
}

${ }^{1}$ Global Immunization Division, Centers for Disease Control and Prevention, Atlanta, Georgia; ${ }^{2}$ National Public Health Laboratory, Ministry of Public Health and Population, Port-au-Prince, Haiti; ${ }^{3}$ Oak Ridge Institute for Science and Education, Centers for Disease Control and Prevention, Atlanta, Georgia; ${ }^{4}$ Division of Parasitic Diseases and Malaria, Centers for Disease Control and Prevention, Atlanta, Georgia; ${ }^{5}$ Pan American Health Organization, Washington, District of Columbia; ' ${ }^{6}$ Directorate of Epidemiology, Laboratory and Research, Ministry of Public Health and Population, Port-au-Prince, Haiti; ${ }^{7}$ Expanded Program on Immunization, Ministry of Public Health and Population, Port-au-Prince, Haiti; ${ }^{8}$ Centers for Disease Control and Prevention, Port-au-Prince, Haiti; ${ }^{9}$ Institut Haitien de l'Enfance, Pétion-Ville, Haiti; ${ }^{10}$ Division of Foodborne, Waterborne, and Environmental Diseases, Centers for Disease Control and Prevention, Atlanta, Georgia

\begin{abstract}
In Haiti, measles, rubella, and maternal and neonatal tetanus have been eliminated, but a diphtheria outbreak is ongoing as of 2019. We conducted a nationally representative, household-based, two-stage cluster survey among children aged 5-7 years in 2017 to assess progress toward maintenance of control and elimination of selected vaccine-preventable diseases (VPDs). We stratified Haiti into West region (West department, including the capital city) and non-West region (all other departments). We obtained vaccination history and dried blood spots, and measured antibody concentrations to VPDs on a multiplex bead assay. Among 1,146 children, national seropositivity was $83 \%(95 \% \mathrm{Cl}$ : 80-86\%) for tetanus, 83\% (95\% Cl: 81-85\%) for diphtheria, 87\% (95\% Cl: 85-89\%) for measles, and 84\% (95\% Cl: $81-87 \%$ ) for rubella. None of the children had long-term immunity to tetanus or diphtheria (IgG concentration $\geq 1$ international unit/mL). Seropositivity in the West region was lower than that in the non-West region. Vaccination coverage was $68 \%$ (95\% Cl: $61-74 \%)$ for $\geq 3$ doses of tetanus- and diphtheria-containing vaccine (DTP3), $84 \%$ (95\% Cl: 80-87\%) for one dose of measles-rubella vaccine (MR1), and 20\% (95\% Cl: 16-24\%) for MR2. The seroprevalence of measles, rubella, and diphtheria antibodies is lower than population immunity levels needed to prevent disease transmission, particularly in the West region; reintroduction of these diseases could lead to an outbreak. To maintain VPD control and elimination, Haiti should achieve DTP3 and MR2 coverage $\geq 95 \%$, and include tetanus and diphtheria booster doses in the routine immunization schedule.
\end{abstract}

\section{INTRODUCTION}

An essential function of national immunization programs is to routinely estimate vaccination coverage to evaluate performance and assess progress toward and maintenance of vaccine-preventable disease (VPD) control and elimination. Serosurveys measuring antibody responses to VPDs offer an objective measure of population immunity that can be useful to identify immunity gaps, monitor progress toward VPD elimination, investigate causes of disease resurgence, and, in certain settings, estimate vaccination coverage. ${ }^{1-3}$ The immune response to vaccination depends on several factors, including the individual's humoral and cellular immune system, age at vaccination, type of vaccine (live versus inactivated), and number of doses received. ${ }^{1,4}$

Haiti's national immunization program has made significant progress toward the Pan American Health Organization (PAHO) regional VPD elimination goals. The last confirmed measles and rubella cases occurred in 2001 and 2006, respectively, ${ }^{5}$ and measles and rubella/congenital rubella syndromes were verified to be eliminated in $2014 .{ }^{6}$ Maternal and neonatal tetanus were verified to be eliminated in 2017. ${ }^{7,8}$ However, a diphtheria outbreak started in Haiti in $2014^{9}$ and continues as of December 2019. ${ }^{10,11}$ In addition, Haiti has not reached the vaccination coverage targets recommended by the PAHO. ${ }^{12,13}$

*Address correspondence to Anna A. Minta, Centers for Disease Control and Prevention, 1600 Clifton Rd., MS H24-3, Atlanta, GA 30329. E-mail: aminta@cdc.gov
Three doses of diphtheria-tetanus-pertussis containing vaccine (DTP) at 6, 10, and 14 weeks, and one dose of measlesrubella vaccine (MR) at 9 months are recommended in the routine vaccination schedule. Diphtheria toxoid and tetanus toxoid are the antigens of interest in this survey. Measles-rubella vaccination campaigns targeting children aged 9 months to 9 years and 9-59 months were conducted in 2012 and 2016 , respectively. ${ }^{6} \mathrm{~A}$ second dose of MR at 13 months of age was introduced in $2016 .{ }^{14}$ A booster dose of DTP has been recommended for children aged 15 months since the 1980s, except 2012-2015 because of financial constraints.

Data on measles, rubella, diphtheria, and tetanus immunity among children in Haiti are unknown and are needed to evaluate progress toward maintaining elimination and preventing epidemics. We conducted this survey to estimate immunity to these VPDs among children aged 5-7 years at the time of the survey. We compared serologic results with survey estimates of DTP and MR coverage.

\section{MATERIALS AND METHODS}

Design and sampling. The survey was a nationally representative, household-based, stratified, two-stage cluster survey conducted in November 2017. The survey was designed to estimate chronic hepatitis B virus (HBV) infection and immunity to diphtheria, tetanus, measles, and rubella. Haiti was stratified into West region, which contains the West department (including the urban metropolitan Port-au-Prince, where $1 / 3$ of Haiti's population lives), ${ }^{15}$ and non-West region (all other departments). The primary and secondary sampling units were 78 enumeration areas (EAs) per region, selected by 
probability proportional to size, and 62 households per EA, selected by simple random sampling. A total of 4,836 households were targeted with the goal to recruit at least 455 children aged 5-7 years per region. For EAs containing 62 or fewer households, all households were included in the survey. During the survey, the teams conducted two revisits to a household before determining that a household was unoccupied or that an eligible child did not live in the household. If more than one eligible child was present, then one child was selected by simple random sampling. Children were included in the survey if the caregiver gave consent for the child's participation. Further details of age selection, sampling, and the sample size calculation, which were based on the estimated HBV infection prevalence, are reported elsewhere. ${ }^{16}$

Data collection. A questionnaire was administered to caregivers, which included questions about household composition, caregiver's demographics and socioeconomic characteristics, child's demographics and education level, and child's vaccination status. Caregivers were asked to present all cards from routine vaccination visits and campaigns.

At least three drops of blood were collected by finger prick onto TropBio filter wheels (Cellabs, Sydney, Australia). The blood spots were dried in the field (creating dried blood spots [DBS]), and then transported to the National Public Health Laboratory in Port-au-Prince, where they were stored in the freezer at $-40^{\circ} \mathrm{C}$. Dried blood spots were analyzed at the CDC Atlanta.

Laboratory methods and analysis. Corynebacterium diphtheriae toxoid (List Biological Laboratories, Campbell, $\mathrm{CA}$ ), tetanus toxoid (Massachusetts Biological Laboratories, Boston, $\mathrm{MA}$ ), and recombinant measles virus $\mathrm{N}(\mathrm{MVN})$ protein (Meridian Life Sciences, Memphis, TN) were purchased from the indicated sources. Recombinant MVN protein from insect cell expression was partially purified by MonoQ HR5/5 column chromatography (GE Healthcare, Piscataway, NJ) in buffer containing $25 \mathrm{mM}$ Tris at $\mathrm{pH}$ 8.0. Measles virus $\mathrm{N}$ protein was eluted from the column between 0.5 and $1.0 \mathrm{M} \mathrm{NaCl}$ and was dialyzed (Spectra Por-3 tubing, Spectrum Laboratories, Rancho Dominguez, CA) into buffer containing $10 \mathrm{mM}$ $\mathrm{NaHPO}_{4}$ at $\mathrm{pH} 7.2$ with $0.85 \% \mathrm{NaCl}$ (phosphate-buffered saline [PBS]). Bead coupling conditions have been described elsewhere. ${ }^{17,18}$ Because the magnetic beads are larger than the previously used SeroMap beads, the protein amounts were increased by $15 \% ; 14.4 \mu \mathrm{g}$ of tetanus toxoid, $69 \mu \mathrm{g}$ of diphtheria toxoid, and $6.9 \mu \mathrm{g}$ of measles protein were used to couple $12.5 \times 10^{6}$ magnetic beads in a $1-\mathrm{mL}$ final volume. Inactivated whole rubella virus (Meridian Life Sciences, grade 4) was coupled using $34.5 \mu \mathrm{g}$ for $12.5 \times 10^{6}$ magnetic beads in $1 \mathrm{~mL}$ of buffer containing $50 \mathrm{mM} 2-(\mathrm{N}$-morpholino)ethanesulfonic acid at $\mathrm{pH} 5.0 \%$ and $0.85 \% \mathrm{NaCl}$.

For each specimen, a single 6-mm punch of DBS was eluted in buffer $\mathrm{B}$ (PBS containing $0.5 \%$ bovine serum albumin [BSA], $0.3 \%$ Tween- $20,0.1 \%$ casein, $0.02 \%$ sodium azide, $0.5 \%$ polyvinyl alcohol, $0.8 \%$ polyvinylpyrrolidone, and $3 \mu \mathrm{g} / \mathrm{mL}$ E. coli extract) by incubation overnight at $4{ }^{\circ} \mathrm{C}$. A final dilution of 1:200 whole blood (equivalent to 1:400 serum dilution with $50 \%$ hematocrit assumption in whole blood) was prepared in buffer $\mathrm{B}$, and specimens were stored at $4^{\circ} \mathrm{C}$ until analysis.

For the detection of $\operatorname{lgG}$ antibodies against the antigens coupled to microbeads, the multiplex bead assay (MBA) was performed as described previously ${ }^{19}$ in flat bottom Bio-Plex Pro 96-well plates (Bio-Rad, Hercules, CA), with specimens run in duplicate. Washes between incubation steps used a handheld magnet (Luminex Corp, Austin, TX). Beads $(250,000$ beads/antigen/plate) were suspended in buffer A (PBS, 0.5\% BSA, $0.05 \%$ Tween-20, and $0.02 \% \mathrm{NaN}_{3}$ ), and $50 \mu \mathrm{L}$ of bead mastermix was added to each well. After addition of $200 \mu \mathrm{L}$ wash buffer (PBS, 0.05\% Tween-20; [PBST]) to each well, wash buffer was left in each well for one minute to allow bead magnetization before inverting the plate to evacuate the wells of liquid. Plates were washed two times with PBST, and $50 \mu \mathrm{L}$ of specimen (at 1:200 whole blood dilution) was added to each well and incubated with shaking at room temperature for 90 minutes. After three washes with PBST, beads were incubated with biotinylated antihuman IgG (1:500, Southern Biotech, Birmingham, $A L)$ and biotinylated antihuman $\operatorname{lgG}_{4}(1: 625$, Southern Biotech). Then, plates were incubated for 45 minutes and washed three times with PBST. Streptavidin conjugated to phycoerythrin (PE) (1:200, Invitrogen, Waltham, MA) was added to detect bound secondary antibody. After a 30-minute incubation, wells were washed three times with PBST and incubated in buffer A for 30 minutes under light shaking to remove any loosely bound antibodies. Specimens were resuspended in $100 \mu \mathrm{L}$ PBS, and fluorescence data were collected immediately on the MAGPIX with Bio-Plex Manag$\mathrm{er}^{\mathrm{TM}}$ software 6.1 (Bio-Rad, Hercules, CA) with a target of 50 beads per region per well. Median fluorescence intensity (MFI) signal was generated for a minimum of 50 beads/region, and background MFI from wells incubated with buffer B was subtracted from each specimen to give a final value of MFI minus background (MFI-bg) for analysis.

Serial dilutions of reference serum standards from the WHO International Laboratory for Biological Standards were made for tetanus (TE-3; 120 international units $/ \mathrm{mL}$ [IU/mL]), rubella (67/182; $80 \mathrm{IU} / \mathrm{mL})$, and diphtheria (10/262; $2 \mathrm{IU} / \mathrm{mL})$. These dilution series were run under the same assay conditions listed earlier and on the same MAGPIX machine as the unknown specimens. Regression curves for each standard were produced to translate an unknown specimen's MFI-bg assay signal value to an IgG concentration of IU/mL serum. A reference standard for the measles virus plaque reduction neutralization test $\left(\mathrm{PRNT}^{20} ; 3 \mathrm{lU} / \mathrm{mL}\right)$ is available for the quantitation of total virus-neutralizing antibody responses, but the standard has not been calibrated for use in ELISA format. Because only lgG antibodies to the MVN protein are detected in our multiplex assay, a ROC-optimized MFI-bg cutoff value that provided good sensitivity and specificity compared with the "gold standard" PRNT was determined (Coughlin et al., unpublished). ${ }^{18}$ This value was translated to the magnetic bead set used in this work and was determined to be $314 \mathrm{MFI}-$ bg units.

Seropositivity for tetanus, diphtheria, and rubella antibodies was defined by extrapolated $\mathrm{IU} / \mathrm{mL}$ antibody concentration. Tetanus and diphtheria antibody seropositivities were defined using the standard cutoff of $0.01 \mathrm{IU} / \mathrm{mL} .^{21-24}$ Tetanus and diphtheria antibody seropositivities were further categorized as $\operatorname{lgG}<0.01 \mathrm{lU} / \mathrm{mL}, 0.01$ to $<0.1 \mathrm{IU} / \mathrm{mL}, 0.10$ to $<1 \mathrm{lU} / \mathrm{mL}$, and $\geq 1$ $\mathrm{IU} / \mathrm{mL}{ }^{17,23}$ These categories are associated with a lack of protection, minimal protection, full protection, and long-term protection against diphtheria. ${ }^{21}$ Increasing antibody concentrations against tetanus are associated with a decreased risk of infection and longer term protection, ${ }^{22}$ and tetanus antibody concentrations $\geq 1 \mathrm{lU} / \mathrm{mL}$ are associated with long-term protection. ${ }^{25,26}$ Rubella antibody seropositivity was defined 
as $\geq 10 \mathrm{IU} / \mathrm{mL} .{ }^{27}$ Measles antibody seropositivity was defined as $314 \mathrm{MFI}-\mathrm{bg}$ units, as described previously.

Statistical analysis. Data from the questionnaires were entered into a Microsoft Access (Microsoft Corp., Redmond, WA) database and analyzed using SAS 9.4 (The SAS Institute, Cary, NC). Descriptive analyses of demographic and socioeconomic characteristics were calculated. Seroprevalence and vaccination coverage estimates, both nationally and for the West and non-West regions, were calculated, accounting for survey design, which included strata, cluster, and weight statements. The SAS statements and survey weight calculations used in this survey are described elsewhere. ${ }^{16}$ The number of card-documented doses and dates of vaccination (if noted on card) with any diphtheria-tetanus-pertussis containing vaccine (DTP) and MR was noted. Diphtheriatetanus-pertussis containing vaccine was recorded as DTP1-4+ (DTP doses 1-4 or more), and MR coverage from routine or campaign cards was combined and recorded as MR1 and MR2+. For children without a card, caregiver recall was used to determine vaccination status. If the caregiver answered "I don't know" to whether the child received any doses or to the number of doses, the child was coded as missing vaccination data. Vaccination coverage estimates by card documentation and caregiver recall were combined to determine coverage by either source.

Diphtheria-tetanus-pertussis containing vaccine $3+$ and MR2+ were considered fully vaccinated because the children had been eligible for three doses of DTP through the routine system and two doses of MR through the routine system plus campaigns. The median time to vaccination was assessed using survival analysis among children with dates on their routine vaccination card, and results were displayed with Kaplan-Meier curves, highlighting timely vaccination (within 2 weeks for DTP1 and DTP3 and 1 month for MR1). Measles-rubella vaccine 2 timeliness was not calculated because MR2 was introduced into the routine immunization program in 2016.

Rao-Scott chi-square tests were used to determine associations between demographic and socioeconomic factors with antibody seropositivity and vaccination coverage, and to compare vaccination coverage to antibody seropositivity. A $P$-value of $<0.05$ was considered statistically significant for all tests.

Ethics/human subjects. The National Bioethics Committee in Haiti and the PAHO Ethics Review Committee reviewed and approved the protocol. The Human Subjects Protection Office at the CDC reviewed the protocol, and the protocol did not require review by the Institutional Review Board.

\section{RESULTS}

General characteristics. During the household enumeration, some EAs contained fewer than 62 households, so a total of 4,736 households were identified for inclusion in the survey. Of 4,736 households, contact was made at 4,587 (97\%) households during the survey, and 1,181 households (26\%) of the 4,587 households included at least one child aged 5-7 years. Among 1,181 children identified for potential enrollment, 1,152 (98\%) caregivers provided consent. Of the 1,152 children included in the survey, 1,146 (99\%) had enough DBS specimen to be included in the analyses. Of the 1,146 children included in the survey, 524 (46\%) lived in the West region and $622(54 \%)$ lived in the non-West region. Among children, 50\% were male and $62 \%$ were in primary school. Among the children's caregivers, $75 \%$ had a job and $33 \%$ had at least some secondary school education (Table 1). Characteristics of the children and their caregivers were similar by region, except that caregivers in the West region had a higher education level than caregivers in the non-West region $(P=0.01)$.

Seropositivity. Nationally, the weighted seropositivity among children aged 5-7 years in Haiti in 2017 was 83\% (95\% Cl: 81-85\%) for diphtheria antibody, 83\% (95\% Cl: 80-86\%) for tetanus antibody, $84 \%$ (95\% Cl: $81-87 \%)$ for rubella antibody, and $87 \%$ (95\% Cl: $85-89 \%)$ for measles antibody (Table 2). Seropositivity for diphtheria, tetanus, and measles antibodies was significantly lower in the West region than in the non-West Region. Seropositivity against rubella was lower in the West region, but the difference was not statistically significant (Table 2). None of the enrolled children had tetanus or diphtheria IgG concentrations $\geq 1.0 \mathrm{IU} / \mathrm{mL}$ (Table 2). When comparing antibody seropositivity with each component of the combination vaccines, $8 \%(95 \% \mathrm{Cl}: 6-11 \%)$ of children were immune to tetanus only, $9 \%(95 \% \mathrm{Cl}$ : 7-11\%) were immune to diphtheria only, $75 \%$ (95\% Cl: 71-77\%) were immune to both, and $9 \%(95 \% \mathrm{Cl}$ : 7-11\%) were susceptible to both. Furthermore, $6 \%$ (95\% Cl: 5-9\%) of children were immune to measles only, 3\% (95 Cl: 2-4\%) were immune to rubella only, $81 \%$ (95\% Cl: $78-84 \%)$ were immune to both, and 10\% (95\% Cl: 8-12\%) were susceptible to both.

Vaccination coverage. Of 1,146 enrolled children, 552 (49\%) had a routine vaccination card available, 145 (13\%) had a campaign card from the 2012 and/or 2016 campaigns, and 603 (54\%) had either a routine or campaign card. There was no difference among children from the West and non-West regions regarding card availability $(P=$ 0.8 ). Few children had both routine and campaign cards (94/ $1,146,8 \%)$. For children without vaccination cards, the caregivers were most likely to report that the card was in another location (48\%) or that the caregiver lost the card (43\%). Diphtheria-tetanus-pertussis containing vaccine or MR status was unknown for 186 (16\%) and 177 (15\%) participants, respectively. Diphtheria-tetanus-pertussis vaccine coverage by card or recall was as follows: DTP1 $91 \%$ (95\% Cl: 87-94\%), DTP2 84\% (95\% Cl: 78-88\%), DTP3 68\% (95\% Cl: 61-74\%), and DTP4+34\% (95\% Cl: 30-39\%). Measles-rubella vaccine 1 coverage by card or recall was $84 \%$ (95\% Cl: 80-87\%), and MR2+ coverage was 20\% (95\% Cl: $16-24 \%)$. There was no difference in vaccination coverage between the West and non-West regions (Table 3).

Of 552 children with cards, vaccination dates were available for 515 (93\%) for DTP1, 465 (84\%) for DTP3, and 451 (82\%) for MR1. The median time to receipt of DTP1 was 10 weeks, DTP3 was 27 weeks, and MR1 was 12 months (Supplemental Figure 1).

Seropositivity and vaccination coverage. Tetanus and diphtheria antibody seropositivities (83\%) were higher than DTP3+ coverage (68\%) by card or recall. Measles antibody seropositivity $(87 \%)$ was higher than and rubella antibody seropositivity (84\%) was similar to MR1+ coverage $(84 \%)$ by card or recall (Figure 1). The proportion of children who were seropositive for each VPD increased with increasing numbers of vaccination doses by card or recall. Among children who received at least three doses of DTP by card or recall, seropositivity was $88 \%$ for diphtheria antibody and $89 \%$ for tetanus antibody. Among children who received at least two doses of MR by card or recall, seropositivity was $89 \%$ for measles antibody and $87 \%$ for rubella antibody (Table 4). 
TABLE 1

Demographic and socioeconomic characteristics of surveyed children aged 5-7 years and their caregivers-Haiti, 2017

\begin{tabular}{|c|c|c|c|c|c|c|}
\hline \multirow[b]{2}{*}{ Characteristic } & \multicolumn{2}{|c|}{ Total, $N=1,146$} & \multicolumn{2}{|c|}{ West region, $N=524$} & \multicolumn{2}{|c|}{ Non-west region, $N=622$} \\
\hline & $n$ & $\%$ & N & $\%$ & N & $\%$ \\
\hline \multicolumn{7}{|l|}{ Gender of child* } \\
\hline Male & 570 & 49.8 & 269 & 51.4 & 301 & 48.4 \\
\hline Female & 575 & 50.2 & 254 & 48.6 & 321 & 51.6 \\
\hline \multicolumn{7}{|l|}{ Age of child (years) } \\
\hline 5 & 353 & 30.8 & 150 & 28.6 & 203 & 32.6 \\
\hline 6 & 410 & 35.8 & 207 & 39.5 & 203 & 32.6 \\
\hline 7 & 383 & 33.4 & 167 & 31.9 & 216 & 34.7 \\
\hline \multicolumn{7}{|l|}{ Education level of child* } \\
\hline None & 42 & 3.7 & 22 & 4.2 & 20 & 3.2 \\
\hline Kindergarten/preschool & 390 & 34.1 & 188 & 36.0 & 202 & 32.5 \\
\hline Primary & 712 & 62.2 & 312 & 59.8 & 400 & 64.3 \\
\hline \multicolumn{7}{|l|}{ Age of caregiver (years) } \\
\hline$<30$ & 316 & 27.6 & 135 & 25.8 & 181 & 29.1 \\
\hline $30-39$ & 463 & 40.4 & 233 & 44.6 & 230 & 37.0 \\
\hline$\geq 40$ & 366 & 32.0 & 155 & 29.6 & 211 & 33.9 \\
\hline Caregiver has a job* & 853 & 74.6 & 378 & 72.3 & 475 & 76.5 \\
\hline \multicolumn{7}{|l|}{ Education level of caregiver } \\
\hline None & 219 & 19.1 & 78 & 14.9 & 141 & 22.7 \\
\hline Some or completed primary & 451 & 39.4 & 164 & 31.3 & 287 & 46.1 \\
\hline Some secondary & 372 & 32.5 & 207 & 40.0 & 165 & 26.5 \\
\hline Completed secondary or higher & 104 & 9.1 & 75 & 14.3 & 29 & 4.7 \\
\hline
\end{tabular}

A sensitivity analysis was performed among children with cards to investigate whether card coverage would more accurately reflect immunity than recall. Immunity by vaccination status was similar for all antigens whether measured by card only or card and recall. For tetanus immunity, there were only 16 children with cards who had no documentation of tetanus vaccination, and, of these children, 54\% (95\% Cl: 30-76\%) were immune to tetanus (Table 4).

\section{DISCUSSION}

This survey is the first to estimate immunity against diphtheria, tetanus, measles, and rubella among children in Haiti and provides important data for Haiti's immunization program.
None of the children showed evidence of long-term protection against tetanus or diphtheria, which supports the biologic finding that immunity wanes over time in the absence of tetanus and diphtheria booster doses. ${ }^{21,22,28,29}$ Maternal and neonatal tetanus elimination has been achieved in Haiti, but long-term immunity is needed to sustain elimination. The lack of long-term protection is concerning because, according to national coverage surveys, DTP3 coverage in Haiti has fallen from $63 \%$ to $55 \%$ from 2012 to $2017 .{ }^{30}$ According to the World Health Organization-United Nations Children's Fund (WHOUNICEF) vaccination coverage estimates, DTP3 coverage was only $64 \%$ in $2018 .{ }^{12}$ In addition, the vaccination coverage estimates for all antigens in Haiti, such as third-dose polio vaccine $\left(64 \%\right.$ in 2018), ${ }^{12}$ are low, which raises concerns for control and elimination of other VPDs.

TABLE 2

Seropositivity for diphtheria, tetanus, measles, and rubella among children aged 5-7 years-Haiti, 2017*

\begin{tabular}{|c|c|c|c|c|c|c|c|}
\hline \multirow[b]{2}{*}{ Disease (immunity threshold) } & \multicolumn{2}{|c|}{ Total, $\mathrm{N}=1,146$} & \multicolumn{2}{|c|}{ West region, $N=524$} & \multicolumn{2}{|c|}{ Non-west region, $N=622$} & \multirow[b]{2}{*}{$P$-value } \\
\hline & $n$ & $\%(95 \% \mathrm{Cl})$ & $n$ & $\%(95 \% \mathrm{Cl})$ & $n$ & $\%(95 \% \mathrm{Cl})$ & \\
\hline Diphtheria ( $\geq 0.01 \mathrm{IU} / \mathrm{mL}$ ) & 934 & $83.1(80.7-85.4)$ & 390 & $75.1(70.5-79.1)$ & 544 & $87.5(84.5-90.0)$ & $<0.001$ \\
\hline Tetanus ( $\geq 0.01 \mathrm{IU} / \mathrm{mL})$ & 930 & $82.7(79.5-85.5)$ & 396 & 75.7 (69.0-81.3) & 534 & $86.5(83.1-89.4)$ & $<0.01$ \\
\hline Rubella ( $\geq 10 \mathrm{IU} / \mathrm{mL})$ & 943 & $84.0(80.5-86.9)$ & 411 & $79.9(73.8-84.9)$ & 532 & $86.1(81.9-89.4)$ & 0.07 \\
\hline $\begin{array}{l}\text { Measles ( } \geq 314 \text { Median fluorescence } \\
\text { intensity minus background) }\end{array}$ & 975 & $87.4(85.1-89.3)$ & 410 & $80.2(75.1-84.5)$ & 565 & $91.2(88.7-93.2)$ & $<0.001$ \\
\hline \multicolumn{8}{|l|}{ Diphtheria immunity categories (IU/mL)† } \\
\hline$<0.01$ & 212 & $16.9(14.6-19.3)$ & 134 & $24.9(20.9-29.5)$ & 78 & $12.5(10.0-15.5)$ & $<0.001$ \\
\hline 0.01 to $<0.1$ & 559 & $48.4(43.8-52.9)$ & 257 & $48.4(42.5-54.4)$ & 302 & $48.3(42.3-54.4)$ & - \\
\hline 0.1 to $<1$ & 375 & $34.8(30.4-39.4)$ & 133 & $26.6(21.6-32.4)$ & 242 & $39.2(33.3-45.4)$ & - \\
\hline$\geq 1$ & 0 & 0 & 0 & 0 & 0 & 0 & - \\
\hline \multicolumn{8}{|l|}{ Tetanus immunity categories (IU/mL) $†$} \\
\hline$<0.01$ & 216 & $17.3(14.5-20.5)$ & 128 & $24.3(18.7-31.0)$ & 88 & $13.5(10.6-16.9)$ & $<0.01$ \\
\hline 0.01 to $<0.1$ & 373 & $32.6(29.2-36.3)$ & 160 & $31.6(26.8-36.7)$ & 213 & $33.2(28.6-38.2)$ & - \\
\hline$\geq 0.1$ to $<1$ & 557 & $50.1(45.8-54.4)$ & 236 & $44.1(38.4-50.0)$ & 321 & $53.3(47.7-58.9)$ & - \\
\hline$\geq 1$ & 0 & 0 & 0 & 0 & 0 & 0 & - \\
\hline
\end{tabular}

†This section compares the proportion of children in each immunity category between the West and non-West regions. 
TABLE 3

Vaccination coverage by source of information among children aged 5-7 years-Haiti, 2017*

\begin{tabular}{|c|c|c|c|c|c|c|c|}
\hline & \multicolumn{2}{|r|}{ Total } & \multicolumn{2}{|c|}{ West region } & \multicolumn{2}{|c|}{ Non-west region } & \multirow[b]{2}{*}{ P-value } \\
\hline & $n$ & $\%(95 \% \mathrm{Cl})$ & $n$ & $\%(95 \% \mathrm{Cl})$ & $n$ & $\%(95 \% \mathrm{Cl})$ & \\
\hline \multicolumn{8}{|l|}{ DTP1† } \\
\hline Vaccination card & 536 & $57.1(51.1-62.8)$ & 241 & $57.0(47.9-65.6)$ & 295 & $57.1(49.5-64.4)$ & 1.0 \\
\hline Caregiver report & 321 & $33.7(29.4-38.2)$ & 141 & $30.9(25.5-36.8)$ & 180 & $35.1(29.4-41.3)$ & 0.3 \\
\hline Either source & 857 & $90.8(87.0-93.5)$ & 382 & $87.9(81.3-92.3)$ & 475 & $92.2(87.5-95.3)$ & 0.2 \\
\hline \multicolumn{8}{|l|}{ DTP2 } \\
\hline Vaccination card & 512 & $55.3(49.1-61.3)$ & 231 & $55.4(46.6-64.0)$ & 281 & $55.2(47.0-63.0)$ & 1.0 \\
\hline Caregiver report & 263 & $28.3(24.6-32.4)$ & 120 & $27.0(22.0-32.1)$ & 143 & $29.2(24.2-34.7)$ & 0.5 \\
\hline Either source & 775 & 83.6 (78.2-87.8) & 351 & $82.1(74.8-87.7)$ & 424 & $84.3(77.0-89.7)$ & 0.6 \\
\hline \multicolumn{8}{|l|}{ DTP3 } \\
\hline Vaccination card & 480 & $52.0(45.4-58.5)$ & 218 & $51.3(42.2-60.4)$ & 262 & $52.4(43.7-60.9)$ & 0.9 \\
\hline Caregiver report & 153 & $15.5(12.8-18.8)$ & 72 & $15.9(12.2-20.4)$ & 81 & $15.3(11.8-19.7)$ & 0.8 \\
\hline Either source & 633 & $67.5(60.8-73.6)$ & 290 & $67.2(56.1-76.4)$ & 343 & $67.7(59.2-75.2)$ & 0.9 \\
\hline \multicolumn{8}{|l|}{ DTP4+ } \\
\hline Vaccination card & 239 & $25.7(21.6-30.3)$ & 115 & $26.5(20.3-33.9)$ & 124 & $25.2(20.1-31.2)$ & 0.8 \\
\hline Caregiver report & 77 & $8.4(6.2-11.1)$ & 33 & $7.5(5.3-10.7)$ & 44 & $8.8(6.0-12.7)$ & 0.6 \\
\hline Either source & 316 & $34.0(29.7-38.6)$ & 148 & $34.1(27.5-41.4)$ & 168 & $34.0(28.6-40.0)$ & 1.0 \\
\hline \multicolumn{8}{|l|}{ MR1‡ } \\
\hline Vaccination card & 525 & $55.0(50.0-60.0)$ & 236 & $55.7(47.1-64.0)$ & 289 & $54.7(48.4-60.8)$ & 0.8 \\
\hline Caregiver report & 272 & $28.7(25.0-32.8)$ & 110 & $24.5(20.7-28.8)$ & 162 & $30.9(25.6-36.7)$ & 0.07 \\
\hline Either source & 797 & 83.7 (80.0-86.9) & 346 & $80.2(71.6-86.7)$ & 451 & $85.5(81.9-88.6)$ & 0.2 \\
\hline \multicolumn{8}{|l|}{ MR2+ } \\
\hline Vaccination card & 86 & $8.6(6.5-11.4)$ & 45 & $10.4(6.6-16.0)$ & 41 & $7.7(5.3-11.1)$ & 0.3 \\
\hline Caregiver report & 109 & $11.3(8.9-14.3)$ & 48 & $11.4(8.4-15.3)$ & 61 & $11.2(8.1-15.4)$ & 0.9 \\
\hline Either source & 195 & $20.0(16.2-24.1)$ & 93 & $21.8(17.0-27.6)$ & 102 & $18.9(14.2-24.7)$ & 0.4 \\
\hline
\end{tabular}

In this survey, diphtheria immunity among children aged 5-7 years was $83 \%$ overall and $75 \%$ in the West region, which may not have reached the level needed for herd protection $(80-85 \%) .{ }^{31}$ Reviews of diphtheria cases in Haiti found that most cases were unvaccinated or incompletely vaccinated, and school-aged children have been disproportionally affected. ${ }^{9,32}$ In the current survey, $9 \%$ of children were immune to diphtheria and not tetanus, which could mean that they were exposed to the diphtheria toxin through natural infection. In response to the epidemic, the Ministry of Public Health and Population launched a vaccination campaign during March-April 2018, and, 1 month after the first phase of the campaign, the number of diphtheria cases and deaths decreased. ${ }^{33}$ Data from the current survey and the diphtheria outbreak, combined with Haiti's low DTP3 coverage, support the need to achieve DTP3 coverage of 95\%, achieve high coverage of DTP4, and introduce tetanus- and diphtheriacontaining booster doses for children aged 4-7 years and 9-15 years in Haiti's routine schedule as per WHO and PAHO recommendations. ${ }^{13,34}$

For rubella, the national and West region-specific seroprevalence estimates were lower than the 85-87\% minimum levels needed for herd protection. ${ }^{35}$ For measles, the national and regional seroprevalence estimates were lower than the minimum herd protection levels of $89-94 \% .^{36}$ These findings are concerning because the country is at risk of an epidemic if either disease were to be introduced into the country. After the 2012 MR campaign for children aged 1-9 years, a coverage survey found that $31 \%$ of children received their first dose of MR during the campaign, which emphasizes the role of vaccination campaigns in reaching children who were missed during routine vaccination. ${ }^{5}$ Haiti's routine MR1 coverage has declined from $65 \%$ in 2012 to $61 \%$ in 2017 , according to national vaccination coverage surveys. ${ }^{30,37}$ In 2018, MR2 coverage was only $38 \%$ according to WHO-UNICEF vaccination coverage estimates. ${ }^{12}$ The measles and rubella immunity data from this survey support the $\mathrm{PAHO}$ and $\mathrm{WHO}$ recommendations that countries should achieve $\geq 95 \%$ coverage with two doses of MR vaccine through the routine immunization system, and that measles-containing vaccine immunization campaigns may be indicated if the routine system has not reached high coverage. ${ }^{36,38}$ Considering the findings of this survey, it may be useful to conduct an immunization campaign with MR and age-appropriate tetanus- and diphtheria toxoidcontaining vaccine.

The antibody seroprevalence against all four VPDs was lower in the West region than in the non-West region. Vaccination coverage surveys have shown lower coverage from routine immunization services and campaigns in the West department/Port-au-Prince area, ${ }^{5,30,37}$ which could be due to difficulties reaching children in urban areas. ${ }^{39,40}$ Before measles elimination, an international measles importation led to an outbreak in Haiti, particularly in Port-au-Prince, ${ }^{5}$ which is further evidence that the West department is at risk of an epidemic. The immunity gap in the West region suggests that additional interventions should take place to improve vaccination coverage in Port-au-Prince.

This serosurvey highlighted benefits of using serosurvey data in addition to vaccination coverage data. Diphtheriatetanus-pertussis containing vaccine 3 coverage by card or recall underestimated seropositivity (83\%) for diphtheria and tetanus antibodies. Some children who did not receive any doses of DTP based on card documentation were immune to tetanus, which could only occur after vaccination. ${ }^{22}$ Surveys 


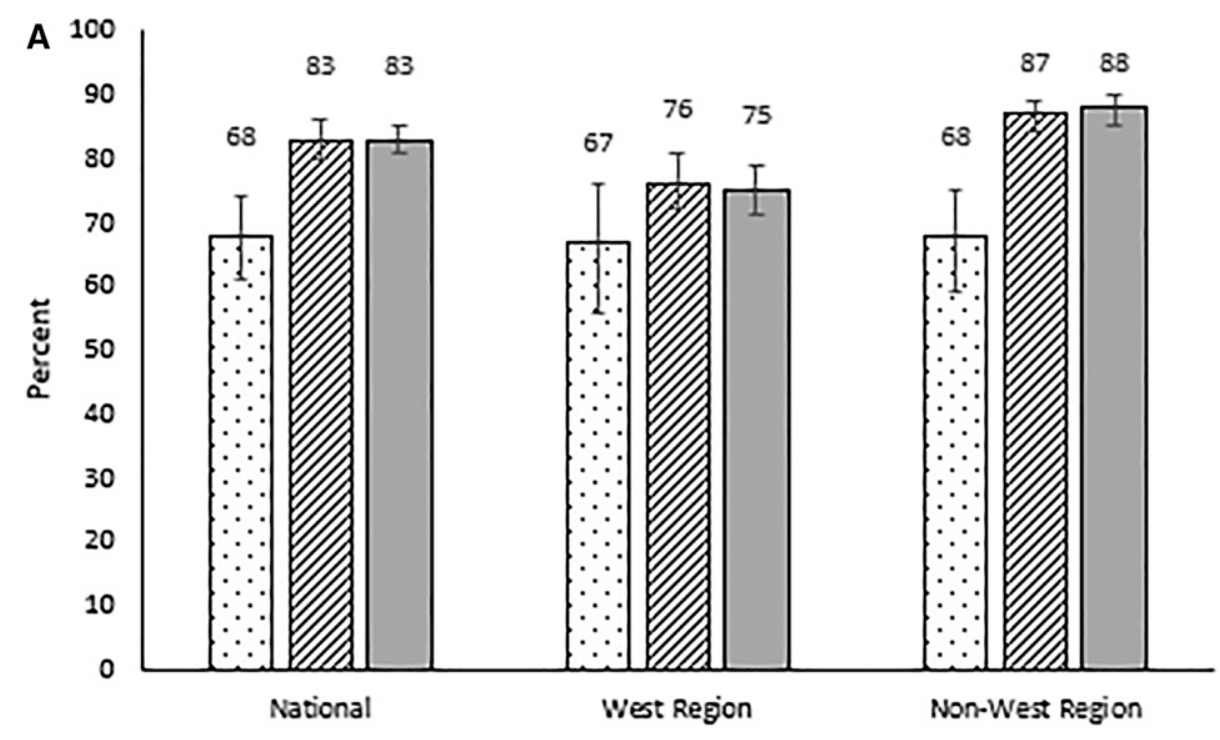

DOTP3+ coverage by card or recall

DTetanus Seropositive (20.01 IU/mL tetanus toxoid IgG)

口Diphtheria Seropositive (20.01 IU/mL diptheria IgG)

\section{B}

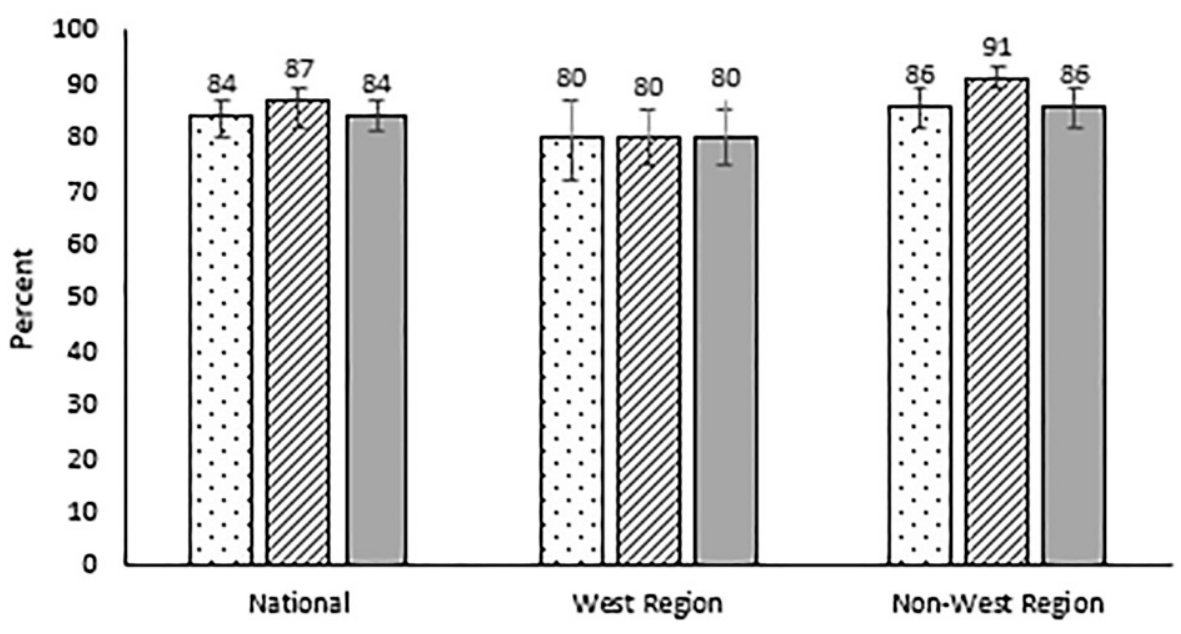

314

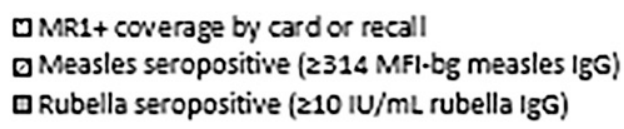

DMR1+ coverage by card or recall

口 Measles seropositive (2314 MFl-bg measles IgG)

a Rubella seropositive ( $210 \mathrm{IU} / \mathrm{mL}$ rubella IgG)

FIGURE 1. Vaccination coverage and seropositivity among children aged 5-7 years old-Haiti, 2017. Numbers represent point estimates, brackets represent $95 \% \mathrm{Cl}$. Tetanus and diphtheria vaccination coverage and seropositivity among children aged 5-7 years-Haiti, 2017. (B) Measles and rubella vaccination coverage and seropositivity among children aged 5-7 years-Haiti, 2017.

using card documentation or recall to assess vaccination coverage are at risk of information and recall bias, and both card documentation and recall have been found to underestimate actual DTP coverage or immunity. ${ }^{17,41,42}$ In addition, almost $20 \%$ of children were excluded from the vaccination coverage estimates because they were missing vaccination cards, and their caregiver did not remember whether the child had been vaccinated. Many of these children might have been vaccinated, leading to higher seroprevalence than vaccination coverage. In this survey, $8 \%$ of children were immune to tetanus and not diphtheria, which indicates that the children were most likely vaccinated against diphtheria and tetanus, but their diphtheria immunity had already waned by the time of the survey. Measles-rubella vaccine $2+$ coverage was likely underestimated in the current survey because all children were eligible for at least two MR doses through the routine system and at least one of the campaigns. An MR vaccination coverage survey conducted after the 2012 MR campaign estimated 


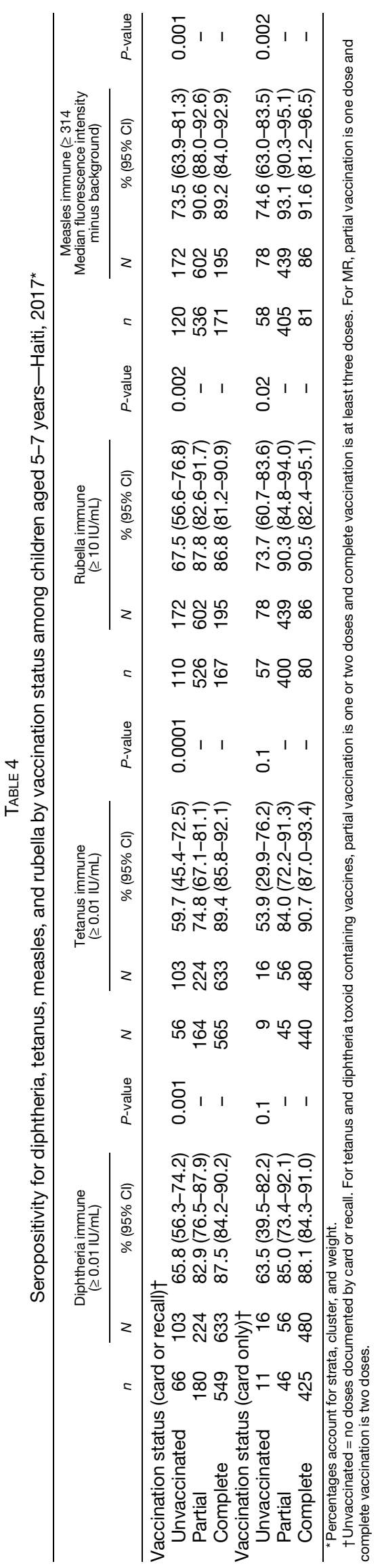

MR2+coverage of $45.7 \%$ (95\% Cl: $41.8-49.7 \%)$ and $27.2 \%$ (95\% Cl: 23.1-31.2\%) for children born in 2010 and 2011, respectively, ${ }^{5}$ compared with $20 \% \mathrm{MR} 2$ + coverage in the current survey, which included children born in those years. In addition, the 2016 MR campaign had $99 \%$ coverage based on administrative data. ${ }^{43}$ The difference in coverage between the different surveys and seroprevalence data is likely due to the low card retention in the current survey and decreasing caregiver recall over time.

There are some limitations if serosurveys are used without vaccination coverage surveys. For diphtheria, measles, and rubella, immunity reflects either vaccination or previous infection; therefore, seroprevalence data alone cannot distinguish these scenarios. ${ }^{4}$ Antibody seroprevalence does not necessarily correlate with the number of doses of vaccines received, ${ }^{1}$ or vaccine timeliness, which are important indicators for the immunization program. In this survey, the median time to DTP3 and MR was 13 weeks and 3 months later, respectively, than recommended, which is similar to findings from a previous vaccination coverage survey in Haiti. ${ }^{44}$ It is also possible that the MBA could detect non-neutralizing antibodies to diphtheria; however, studies have shown that the MBA performs well compared with the gold standard Vero cell test. ${ }^{45,46}$

This survey provides the first estimates of immunity to diphtheria, tetanus, measles, and rubella among children in Haiti. The findings highlight the need to attain $\geq 95 \%$ DTP3 and MR2 coverage nationally, particularly in the metropolitan Portau-Prince area, and to introduce tetanus- and diphtheria toxoid-containing booster doses for children aged 4-7 years and 9-15 years and sustain high coverage of booster doses. The survey results also highlight the benefit of supplementing vaccination coverage data with seroprevalence data to provide a better assessment of the vaccination program and identify immunity gaps. Without achieving high vaccination coverage and adequate booster doses as per the WHOrecommended schedule, Haiti risks reestablishment of measles or rubella, an increase in maternal and neonatal tetanus cases, and ongoing diphtheria epidemics.

Received February 10, 2020. Accepted for publication June 1, 2020.

Published online July 6, 2020.

Note: Supplemental figure appears at www.ajtmh.org.

Acknowledgments: We thank Paule-Andrée Louis-Byron, Maxi Raphael, and Dieula Louissaint, National Coordination Unit of the Vaccination Program, Haiti; Patrick Dely and Annemarie Desormaux, Directorate of Epidemiology, Laboratory and Research, Haiti; Alexandre Existe and Jacquelin Presume, National Public Health Laboratory, Ministry of Public Health and Population, Haiti; Jean Andre, PAHO Haiti; Alexandre Canez, Michaud Josue, Vilus Haydn Joseph Lutherson, Louis Jean Gerlan, Ardiles David Brignol, Stephania Noel, Myriam Israel, Jean Albertiny Calixte Chery, Moselande Pierre, Benjamin Joseph, Kettia Negaud, Nardine Massillon, Jeannette Georges, Sophia Laurore Bresemy, Edelyne Claude, Geraldine Loubeau, Saraphyna Brunvil, Smith Gustave, Gerardine Gerard, Guersley Chery, Guilene Estesias, Florene Saintelien, James Dodo, Eltha Tanis, Jean Claude St Fleur, Marie Sonide Dorilma, Nathalie Cadet Abraham, Ghislaine Romain, Marie Elisna Eliscat, Jean Patrick Gerard, Sherly Gay, Emie Gabriel, Makenley Moise, Hermine Michel, Johnson Mervil, Nathalie Laguerre, Fabienne Cerelus Mildor, Jean Renand Barreau, Kettie Parfait, Josue Dormelus, Marie Emmanuelle Casimir Philogene, Ashley Desormes, Valex Paul, Oudeline Mele, Josue Pierre, Marie Myrtha Ferjuste, Jean Roberto Target, Nathalie Louis, Wislene Noel, Kesnel Mogelin, Ange Laure Duclaire, Marie Guerda Etienne, Sophia Vales, Clenise Thermitus, Marie Yolande Medor, Jenita Cius, Joanne Joseph, Alence Therone, Jeanise Jean, Mirlene Laurenceau, Igline Dorilas, Jimmy Ezean, Kerline Floritan, 
Guerna Aldeus, Saul Junior Estesias, Cathelie Menelas, Alexis Felix, Catiana Felix, and Modeline Francois, Institut Haïtien de l'Enfance, Haiti; Kathleen Wannemuehler, Amber Dismer, Gretchen Cooley, and Elisabeth Krow-Lucal, CDC.

Financial support: This survey was supported by a cooperative agreement between the CDC and the Haiti Ministry of Public Health and Population and a cooperative agreement between the CDC and PAHO.

Disclosures: Valery Blot works for Institut Haitien de l'Enfance, the institution that was funded through a cooperative agreement between CDC and the Haiti Ministry of Health and Population to implement the survey. The findings of this survey were presented in part at the IDWeek 2019 conference October 2-6, 2019, Washington, DC.

Disclaimer: The findings and conclusions in this article are those of the authors and do not necessarily reflect the position of the Centers for Disease Control and Prevention nor the Pan American Health Organization. Abstract: A. A. Minta, J. Andre-Alboth, L. Childs, D. Nace, G. Rey-Benito, J. Boncy, P. Adrien, J. François, N. Charles, V. Blot, J. Vanden Eng, J. W. Priest, E. Rogier, R. A. Tohme. Seroprotection against Measles, Rubella, Tetanus, and Diphtheria Among Children in Haiti-2017. Open Forum Infectious Diseases, Volume 6, Issue Supplement_2, October 2019, Pages S977-S978, https://doi.org/ 10.1093/ofid/ofz360.2448 (accessed November 4, 2019).

Authors' addresses: Anna A. Minta, Lana Childs, Douglas Nace, Jodi Vanden Eng, Jeffrey W. Priest, Eric Rogier, and Rania A. Tohme, Centers for Disease Control and Prevention, Atlanta, GA, E-mails: aminta@ cdc.gov, Ichilds44@gmail.com, ddn4@cdc.gov, jodi.vandeneng@ gmail.com, jip8@cdc.gov, and inb1@cdc.gov. Gloria Rey-Benito, Pan American Health Organization, Washington, DC, E-mail: reyglori@ paho.org. Jocelyne Andre-Alboth, Jacques Boncy, Paul Adrien, and Jeannot Francois, Ministry of Public Health and Population, Port-auPrince, Haiti, E-mails: alboth28@yahoo.fr, jboncy2001@yahoo.fr, padrien2004@yahoo.fr, and francoisjeannot@yahoo.fr. Valery Blot, Institut Haitien de L'Enfance, Petion-Ville, Haiti, E-mail: valeryblot@ yahoo.fr.

\section{REFERENCES}

1. Cutts FT, Hanson M, 2016. Seroepidemiology: an underused tool for designing and monitoring vaccination programmes in lowand middle-income countries. Trop Med Int Health 21: 1086-1098.

2. Winter AK, Martinez ME, Cutts FT, Moss WJ, Ferrari MJ, McKee A, Lessler J, Hayford K, Wallinga J, Metcalf CJE, 2018. Benefits and challenges in using seroprevalence data to inform models for measles and rubella elimination. $J$ Infect Dis 218: 355-364.

3. World Health Organization (WHO), 2019. SAGE Working Group on Quality and Use of Immunization and Surveillance Data: Executive Summary. Geneva, Switzerland: WHO.

4. MacNeil A, Lee CW, Dietz V, 2014. Issues and considerations in the use of serologic biomarkers for classifying vaccination history in household surveys. Vaccine 32: 4893-4900.

5. Tohme RA et al., 2014. Measles and rubella vaccination coverage in Haiti, 2012: progress towards verifying and challenges to maintaining measles and rubella elimination. Trop Med Int Health 19: 1105-1115.

6. Tohme RA et al., 2017. Expansion of vaccination services and strengthening vaccine-preventable diseases surveillance in Haiti, 2010-2016. Am J Trop Med Hyg 97: 28-36.

7. Pan American Health Organization (PAHO), 2018. Immunization Newsletter. Washington, DC: PAHO/WHO.

8. UNICEF, 2017. Region of the Americas Eliminates Maternal and Neonatal Tetanus. Washington, DC and New York, NY: UNICEF.

9. Clerville J, Delmas HT, 2018. Diphtheria outbreak, Haiti, 2014-2017: an epidemiological profile and A case fatality rate trend analysis (scientific poster). Int Soc Infect Dis 73: 274-275.

10. Pan American Health Organization (PAHO), 2019. Epidemiological Update: Diphtheria. Washington, DC: PAHO/WHO.

11. Pan American Health Organization (PAHO), 2020. Epidemiological Update: Diphtheria. Washington, DC: PAHO/WHO.
12. World Health Organization, 2019. WHO Vaccine-Preventable Diseases: Monitoring System. 2019 Global Summary. Available at: http://apps.who.int/immunization_monitoring/globalsummary/ countries?countrycriteria\%5Bcountry\%5D\%5B\%5D=HTI. Accessed April 29, 2020.

13. Pan American Health Organization (PAHO), 2015. Plan of Action on Immunization. Washington, DC: PAHO.

14. Dabbagh A, Patel MK, Dumolard L, Gacic-Dobo M, Mulders MN, Okwo-Bele JM, Kretsinger K, Papania MJ, Rota PA, Goodson $\mathrm{JL}$, 2017. Progress toward regional measles eliminationworldwide, 2000-2016. MMWR Morb Mortal Wkly Rep 66: 1148-1153.

15. Institut Haïtien de Statistique et D'Informatique, 2015. Population Totale, Population de 18 Ans et Plus: Menages et Densites Estimes en 2015. Port-au-Prince, Haiti: IHSI.

16. Childs L, Adrien P, Minta AA, Francois J, Phaimyr Jn Charles N, Blot V, Rey-Benito G, Vanden Eng JL, Tohme RA, 2019. Prevalence of chronic hepatitis $B$ virus infection among children in Haiti, 2017. Am J Trop Med Hyg 101: 214-219.

17. Scobie HM et al., 2016. Tetanus immunity among women aged 15 to 39 Years in Cambodia: a national population-based serosurvey, 2012. Clin Vaccine Immunol 23: 546-554.

18. Ondigo BN et al., 2018. Impact of mothers' schistosomiasis status during gestation on children's IgG antibody responses to routine vaccines 2 years later and anti-schistosome and antimalarial responses by neonates in western Kenya. Front Immunol 9: 1402.

19. Rogier E, Moss DM, Chard AN, Trinies V, Doumbia S, Freeman MC, Lammie PJ, 2017. Evaluation of immunoglobulin G responses to Plasmodium falciparum and Plasmodium vivax in Malian school children using multiplex bead assay. Am J Trop Med Hyg 96: 312-318.

20. National Institute for Biological Standards and Control, 2008. WHO International Standard 3rd International Standard for Antimeasles. Available at: https://www.nibsc.org/documents/ifu/ 97-648.pdf. Accessed April 29, 2020.

21. Tiwari TSP, Wharton M, 2018. Diphtheria toxoid. Plotkin S, Orenstein W, Offit P, Edwards K, eds. Vaccines. Philadelphia, PA: Elsevier, 261-275.e7.

22. Roper M, Wassilak S, Scobie H, Ridpath A, Orenstein W, 2018. Tetanus toxoid. Plotkin S, Orenstein W, Offit P, Edwards K, eds. Vaccines. Philadelphia, PA: Elsevier, 1052-1079.e18.

23. Scobie HM et al., 2017. Tetanus immunity gaps in children 5-14 years and men $>/=15$ years of age revealed by integrated disease serosurveillance in Kenya, Tanzania, and Mozambique. Am J Trop Med Hyg 96: 415-420.

24. Swart EM, van Gageldonk PG, de Melker HE, van der Klis FR, Berbers GA, Mollema L, 2016. Long-term protection against diphtheria in The Netherlands after 50 years of vaccination: results from a seroepidemiological study. PLoS One 11: e0148605.

25. Wagner KS, White JM, Andrews NJ, Borrow R, Stanford E, Newton E, Pebody RG, 2012. Immunity to tetanus and diphtheria in the UK in 2009. Vaccine 30: 7111-7117.

26. Basta NE et al., 2015. Higher tetanus toxoid immunity 2 years after PsA-TT introduction in Mali. Clin Infect Dis 61 (Supp/ 5): S578-S585.

27. Reef S, Plotkin S, 2018. Rubella vaccines. Plotkin S, Orenstein W, Offit P, Edwards K, eds. Vaccines. Philadelphia, PA: Elsevier, 970-1000.e18.

28. Edmunds WJ et al., 2000. The sero-epidemiology of diphtheria in western Europe. ESEN project. European sero-epidemiology network. Epidemiol Infect 125: 113-125.

29. Trollfors B, Knutsson N, Taranger J, Mark A, Bergfors E, Sundh V, Lagergard T, 2006. Diphtheria, tetanus and pertussis antibodies in 10-year-old children before and after a booster dose of three toxoids: implications for the timing of a booster dose. Eur J Pediatr 165: 14-18.

30. Institut Haitien de l'Enfance et ICF, 2018. Enquête Mortalité, Morbidité et Utilisation des Services (EMMUS-VI 2016-2017). Pétion-Ville Haïti, ed. Rockville, MD: IHE et ICF.

31. Anderson RM, 1992. The concept of herd immunity and the design of community-based immunization programmes. Vaccine 10: 928-935. 
32. Exavier MM, Paul Hanna M, Muscadin E, Freishstat RJ, Brisma JP, Canarie MF, 2019. Diphtheria in children in northern Haiti. $J$ Trop Pediatr 65: 183-187.

33. Pan American Health Organization, 2018. Ad hoc TAG Meeting: July 2018. Washington, DC: PAHO/WHO.

34. World Health Organization, 2017. Tetanus vaccines: WHO position paper - February 2017. Weekly Epidemiol Record 92: 53-76.

35. World Health Organization Regional Office for Europe, 2013. Guidance on Conducting Serosurveys in Support of Measles and Rubella Elimination in the WHO European Region. Copenhagen, Denmark: WHO.

36. World Health Organization, 2017. Measles vaccines: WHO position paper - April 2017. Weekly Epidemiol Record 92: 205-228.

37. Cayemittes $M$, Fatuma Busangu M, de Dieu Bizimana J, Barrère $B$, Sévère B, Cayemittes V, Charles E, 2013. Enquête Mortalité, Morbidité et Utilisation des Services, Haïti, 2012. Calverton, MD: MSPP.

38. Pan American Health Organization, 2018. Regional Immunization Plan: Progress Report January-December 2017. Washington, DC: PAHO/WHO.

39. Rainey JJ, Watkins M, Ryman TK, Sandhu P, Bo A, Banerjee K, 2011. Reasons related to non-vaccination and undervaccination of children in low and middle income countries: findings from a systematic review of the published literature, 1999-2009. Vaccine 29: 8215-8221.

40. Nelson KN, Wallace AS, Sodha SV, Daniels D, Dietz V, 2016. Assessing strategies for increasing urban routine immunization coverage of childhood vaccines in low and middle-income countries: a systematic review of peer-reviewed literature. Vaccine 34: 5495-5503

41. Travassos MA et al., 2016. Immunization coverage surveys and linked biomarker serosurveys in three regions in Ethiopia. PLoS One 11: e0149970.

42. Dansereau E, Brown D, Stashko L, Danovaro-Holliday MC, 2019. A systematic review of the agreement of recall, home-based records, facility records, BCG scar, and serology for ascertaining vaccination status in low and middle-income countries. Gates Open Res 3: 923.

43. Ridpath AD, Scobie HM, Shibeshi ME, Yakubu A, Zulu F, Raza AA, Masresha B, Tohme R, 2017. Progress towards achieving and maintaining maternal and neonatal tetanus elimination in the African region. Pan Afr Med J 27: 24.

44. Rainey JJ, Lacapere F, Danovaro-Holliday MC, Mung K, Magloire R, Kananda G, Cadet JR, Lee CE, Chamouillet H, Luman ET, 2012. Vaccination coverage in Haiti: results from the 2009 national survey. Vaccine 30: 1746-1751.

45. van Gageldonk PG, von Hunolstein C, van der Klis FR, Berbers GA, 2011. Improved specificity of a multiplex immunoassay for quantitation of anti-diphtheria toxin antibodies with the use of diphtheria toxoid. Clin Vaccine Immunol 18: 1183-1186.

46. von Hunolstein C, Ralli L, Pinto A, Stickings P, Efstratiou A, Czumbel I, Participants EE, Gaggioli A, 2014. Relevance and criticality in an external quality assessment for the determination of diphtheria antitoxin. J Immunol Clin Res 2. 